\title{
Persistent Model Order Reduction for Complex Dynamical Systems Using Smooth Orthogonal Decomposition
}

\author{
Shahab Ilbeigi David Chelidze * \\ Department of Mechanical, Industrial, and Systems Engineering \\ University of Rhode Island, Kingston, RI 02881 \\ Revised on April 8, 2017
}

\begin{abstract}
Full-scale complex dynamic models are not effective for parametric studies due to the inherent constraints on available computational power and storage resources. A persistent reduced order model (ROM) that is robust, stable, and provides high-fidelity simulations for a relatively wide range of parameters and operating conditions can provide a solution to this problem. The fidelity of a new framework for persistent model order reduction of large and complex dynamical systems is investigated. The framework is validated using several numerical examples including a large linear system and two complex nonlinear systems with material and geometrical nonlinearities. While the framework is used for identifying the robust subspaces obtained from both proper and smooth orthogonal decompositions (POD and SOD, respectively), the results show that SOD outperforms POD in terms of stability, accuracy, and robustness.
\end{abstract}

Keywords: nonlinear model reduction, proper orthogonal decomposition, smooth orthogonal decomposition, complex dynamical system, subspace robustness.

\section{Introduction}

Considerable progress in computing technology in the past few decades did not alleviate difficulty inherent in simulating complex dynamical systems. Examples of such systems are large-scale finite difference/element, multi-body dynamics, or geometrically nonlinear models, and molecular dynamics simulations [1-6]. A reduced order model (ROM) for these systems can be used to significantly reduce redundant computations and data storage requirements [7]. In particular, persistent ROMs, which are robust to the changes in system parameters and loading conditions, can be used in parametric studies that are prohibitive when using a full-scale model. While a variety of methods for model order reduction (MOR) have been developed, very few of them provide persistent ROMs. Often emphasis is only on the accuracy of the ROMs and their ability to capture the dynamics of the full-scale models for a fixed set of parameters, and operating and loading conditions. However, the importance of the robustness of a ROM to the changes in those parameters is often not accentuated. We consider a ROM to be persistent if it is robust to changes in a full-scale model's energy,

*email: chelidze@uri.edu $\diamond$ phone: 401.874.2356 $\diamond$ fax: 401.874.2355 $\diamond$ web: mcise.uri.edu/chelidze/

(C) 2017. This manuscript version is made available under the Elsevier user license http://www.elsevier.com/open-access/userlicense/1.0/ 
forcing, and parameters. Data-based reduced order modeling with no persistency is of limited scope; ROMs built on the data generated from the simulations of a full-scale model can only be used for simulating the same exact configuration of the model. This ROM might still be of great utility if we can study the long-time dynamics of a system (e.g., protein folding), but cannot be utilized in parametric studies, wherein we repeatedly change the parameters, input values, and energy levels.

In this paper, we present a new framework for obtaining persistent ROMs, which are valid within a defined range of the system's energy, which is imposed by changing the input parameters. Our framework can be applied to all data-based MOR methods. We make use of data from simulations or experiments to develop the ROMs. Our goal is to ensure that the obtained persistent ROMs are robust and can be used for simulating the system with any chosen parameter from the defined range.

\subsection{Background and Prior Work}

Persistent MOR for linear systems has not attracted extensive research focus since the linear modal structure is not dependent on the energy of a system. As a result, if a ROM is properly developed for one energy level of a linear system, it should also be valid for the other energy levels. The methodologies for MOR for these systems are mostly projection based, where the linear subspaces used in the projection can be related to the modal space which is spanned by linear normal modes (LNMs). For example, the modes identified using proper orthogonal decomposition (POD) (also known as singular value decomposition, principal component analysis, or Karhunen-Loève expansion) [8-12] approximate the LNMs for systems with uniform mass distribution [13]. Other popular methodologies for the MOR of linear systems include the Galerkin reduction using linear normal modes (LNMs) [14, 15], Krylov subspace projections [16], Hankel norm approximations [17, 18], and truncated balance realizations $[19,20]$.

Nonlinearity, the integral part of complex dynamical systems, makes the development of persistent ROMs a much harder problem. Many approaches for nonlinear MOR are based on extending the methodologies used for linear MOR. For example, linearization about an equilibrium point was used for the reduction of weakly nonlinear systems [21, 22]. Many other approaches are derived from POD $[8,9,11,23-25]$, and some from balanced truncation [26-28]. Some other approaches include neural networks [29], Voltera theory [30], and inertial manifold approximation [31]. More recently, a method called Proper Generalized Decomposition (PGD) has been developed as a generalization of POD in order to construct a priori ROM [32-35]. This method has a potential for solving multidimensional problems since it doesn't require any knowledge of the solution $[34,36]$. The interested reader can find a review on PGD-based MOR techniques in [37].

In summary, a majority of the methodologies commonly used for MOR of nonlinear systems can be categorized into two groups. In the first group, nonlinear normal modes (NNMs) or their approximations [38-44] are used. In the second group, combined with the Galerkin projection, linear subspaces obtained from spatiotemporal decompositions such as POD and smooth orthogonal decomposition (SOD) are utilized $[2,9,10,25,45,46]$. Linear subspaces are of considerable current interest because they are computationally tractable and do not neglect the nonlinearity of the original vector-field [8], while, in general, the calculation of NNMs is difficult [47-50]. Also, MOR based on NNMs suffers from another major drawback related to changes in the NNMs with the variation in system's level of energy $[48,51]$. The dependence of the NNMs on the energy level causes an insufficient robustness of the corresponding NNM-based ROMs to the changes in the system's energy 
level. Thus, NNM-based ROMs cannot be considered truly persistent.

\subsection{Our Approach to Persistent Reduced Order Modeling}

Our approach is based on identifying robust subspaces which do not change drastically as the system changes its energy level. Note that linear subspaces used for MOR are to be identified in such a way that the active NNMs are embedded in them [13]. These subspaces may still change as the NNMs change with the system's level of energy [51]. However, depending on the decomposition method, some particular subspaces may be robust to variations such as changes in initial conditions, external excitations, energy levels, or systems parameters. Our hypothesis is that while an individual NNM may change with energy, a linear subspace embedding this mode may not undergo any considerable change. Identifying such linear subspaces would enable us to obtain the persistent ROMs that are robust to a relatively wide range of system parameters and operating conditions.

The new framework for persistent MOR of large, complex systems based on the concepts of subspace robustness and dynamical consistency is investigated. These concepts have been recently proposed and discussed in our conference presentations [2, 52, 53], where the MOR subspace robustness for a small dynamical system was evaluated. Subspace robustness characterizes how a linear subspace changes under different conditions of the system, which can be used for complex systems to identify the subspace characteristics that lead to a persistent MOR. Dynamical consistency evaluates the deterministic properties of the full-scale system's trajectory projection onto the corresponding linear subspace. It indicates the ability of the identified subspace to potentially — but not necessarily — result in a stable and accurate ROM.

The utility of our framework will be initially evaluated by applying it to the POD subspaces since they are widely used for MOR. POD's drawback for deterministic systems is that it only considers the statistical (i.e., spatial) characteristics of the data [54]. It only prioritizes the maximal variances in the multivariate data and may disregard important dynamical features that have small variances. Changing the energy level of a system may drastically alter dynamic features that previously had small variances, which will not be reflected in the identified POD modal structure. Therefore, POD, while providing an optimal reduction - in the least squares sense - for a system with fixed set of parameters and forcing, might not be a suitable choice for the persistent MOR of complex systems. The subspace obtained from SOD, which was first used in 2005 for vibration mode identification [54], will also be considered within our framework. SOD can be viewed as an extension of POD, which acquires the ability to separate multivariate data based on inherent characteristic frequencies. In other words, it not only considers the spatial statistics, but also looks at the temporal characteristics of data. Thus, SOD subspaces are likely to be less sensitive to the changes in the energy and properties of the system, and may provide for the persistent MOR.

The focus of this study is on complex, nonlinear dynamical systems. However, a lightly damped linear system will be considered first. The rationale behind this consideration is twofold: (1) the assertion that POD recovers LNMs for systems with uniform mass distribution [13] has been only tested on fairly low-dimensional systems, with fairly long time series; and (2) while SOD does not require uniform mass distribution for convergence to the LNMs [54], it has not been tested on large scale systems. Since the LNM structure does not vary with the changes in energy or initial conditions - the corresponding subspaces are robust to these changes - we can use a large-scale linear model to test both the POD and SOD methods' ability to identify LNMs with limited data in different loading scenarios. In addition, we can also evaluate the ability of these methods to provide robust 
subspace identification for a system that actually possesses this robustness in all LNMs.

Following the example of the linear systems, MOR of two large-scale, complex nonlinear systems will be studied as the main subject of this paper. POD and SOD will be used for multivariate analyses of the associated ill-conditioned data matrices from these systems. The POD- and SODspanned subspaces will be tested using the framework to identify the robust subspaces for persistent ROM development. The resultant ROMs subjected to different energy levels will be simulated using several numerical examples. The validity of the results will be investigated in terms of the stability and accuracy of the ROMs.

The rest of this paper is organized as follows. In Section 2, the procedure for projection-based nonlinear model reduction using POD and SOD is reviewed. Section 3 describes the developed framework for the persistent MOR. In Section 4, the full-scale models of one linear and two nonlinear systems are described. Results of the ROM simulations are presented and discussed in Section 5, followed by concluding remarks in Section 6 .

\section{Projection-Based Nonlinear Model Reduction}

We consider a full-scale model of a deterministic dynamical system that has the following form:

$$
\dot{\mathbf{y}}=\mathbf{f}(\mathbf{y}, t),
$$

where $\mathbf{y} \in \mathbb{R}^{2 n}$ is a dynamic state variable, $\mathbf{f}: \mathbb{R}^{2 n} \times \mathbb{R} \rightarrow \mathbb{R}^{2 n}$ is some nonlinear flow, $t$ is time, and $n \in \mathbb{N}$ is the number of the system's degrees of freedom. The state variable trajectory data can be arranged in the matrix $\mathbf{Y}=\left[\mathbf{y}_{1}, \mathbf{y}_{2}, \ldots, \mathbf{y}_{2 n}\right]$. A basis for $\mathbf{Y}$ can be estimated using either the POD or SOD procedures outlined in Ref. [54]. The most dominant $k$-dimensional basis vectors are arranged in the matrix $\mathbf{P}_{k}=\left[\mathbf{e}_{1}, \mathbf{e}_{2}, \ldots, \mathbf{e}_{k}\right]$. The reduced state variable is obtained using a coordinate transformation of $\mathbf{q}=\mathbf{P}_{k} \mathbf{y}$, and the corresponding ROM is:

$$
\dot{\mathbf{q}}=\mathbf{P}_{k}^{\dagger} \mathbf{f}\left(\mathbf{P}_{k} \mathbf{q}, t\right),
$$

where $(.)^{\dagger}$ indicates the pseudoinverse of (.).

\subsection{Proper and Smooth Orthogonal Decomposition}

To build ROMs using the extracted modes from the multivariate analysis, the state variable measurements of the full-scale system are recorded to form position and velocity data matrices $\mathbf{X} \in \mathbb{R}^{r \times n}$ and $\mathbf{V} \in \mathbb{R}^{r \times n}$, respectively. $\mathbf{X}$ is composed of $r$ snapshots of $n$ position state variables. Similarly, $\mathbf{V}$ is composed of $r$ snapshots of $n$ velocity state variables. Thus, the data matrix $\mathbf{Y}$, which we call as full data matrix throughout this paper, is formed by combining $\mathbf{X}$ and $\mathbf{V}$ together, i.e., $\mathbf{Y}=[\mathbf{X} \mathbf{V}]$.

The time derivative of $\mathbf{X}$ is $\mathbf{V}$. To obtain a time derivative of $\mathbf{V}$, or an acceleration data matrix A, we can use a full model of our dynamical system, Eq. (1). Alternatively, it can be approximated by $\mathbf{A} \approx \mathbf{D V}$, where $\mathbf{D}$ is the matrix form of some differential operator such as forward difference. Therefore, an ensemble of time derivative of $\mathbf{Y}$ will be $\dot{\mathbf{Y}}=[\mathbf{V} \mathbf{A}]$. Provided that $\mathbf{Y}$ and $\dot{\mathbf{Y}}$ are zero mean, the corresponding auto-covariance matrices can be formed by

$$
\boldsymbol{\Sigma}_{y y}=\frac{1}{r-1} \mathbf{Y}^{\mathrm{T}} \mathbf{Y}, \quad \boldsymbol{\Sigma}_{\dot{y} \dot{y}}=\frac{1}{r-1} \dot{\mathbf{Y}}^{\mathrm{T}} \dot{\mathbf{Y}} .
$$

In POD, we are looking for a basis vector $\phi \in \mathbb{R}^{2 n}$ such that a projection of the data matrix onto this vector has maximal variance. The solution to the POD problem is achieved by solving the eigenvalue problem of the auto-covariance matrix $\boldsymbol{\Sigma}_{y y}$ in Eq. (3): 


$$
\Sigma_{y y} \phi_{k}=\lambda_{k} \phi_{k},
$$

where $\lambda_{k}$ are proper orthogonal values (POVs), $\phi_{k} \in \mathbb{R}^{2 n}$ are proper orthogonal modes (POMs), and proper orthogonal coordinates (POCs) are columns of $\mathbf{Q}=\mathbf{Y} \boldsymbol{\Phi}$, in which $\mathbf{\Phi}=\left[\phi_{1}, \phi_{2}, \ldots, \phi_{2 n}\right] \in$ $\mathbb{R}^{2 n \times 2 n}$. POVs are ordered such that $\lambda_{1} \geq \lambda_{2} \geq \ldots \geq \lambda_{2 n}$, and reflect the variances in $\mathbf{Y}$ data along the corresponding POMs.

In SOD, we are looking for a basis vector $\psi \in \mathbb{R}^{2 n}$ such that a projection of the data matrix onto this vector has both minimal roughness and maximal variance. The solution to the SOD problem, is achieved by solving a generalized eigenvalue problem of the matrix pair $\boldsymbol{\Sigma}_{y y}$ and $\boldsymbol{\Sigma}_{\dot{y} \dot{y}}$ in Eq. (3):

$$
\boldsymbol{\Sigma}_{y y} \psi_{k}=\lambda_{k} \boldsymbol{\Sigma}_{\dot{y} \dot{y}} \psi_{k},
$$

where $\lambda_{k}$ are smooth orthogonal values (SOVs), $\psi_{k} \in \mathbb{R}^{2 n}$ are smooth projection modes (SPMs), smooth orthogonal modes (SOMs) are $\boldsymbol{\Psi}^{-T}$, and smooth orthogonal coordinates (SOCs) are given by $\mathbf{Q}=\mathbf{Y} \mathbf{\Psi}$, where $\mathbf{\Psi}=\left[\psi_{1}, \psi_{2}, \ldots, \psi_{2 n}\right] \in \mathbb{R}^{2 n \times 2 n}$. The degree of smoothness of the coordinates is described by the magnitude of the corresponding SOV. Thus, the greater in magnitude the SOV, the smoother in time the corresponding coordinate. It should be noted that if we were to replace $\boldsymbol{\Sigma}_{\dot{y} \dot{y}}$ with the identity matrix, the formulation would yield the proper orthogonal decomposition.

\section{Robust Subspace Selection for Persistent MOR}

The appropriate subspace for model reduction can be selected based on a newly developed criteria [53]. These criteria quantifies two concepts: dynamical consistency-which demonstrates how well the linear subspace embeds the nonlinear manifold, and subspace robustness - which explains the sensitivity of the subspace to changes in the system's level of energy. Here, quantifications of these concepts are briefly restated. A more complete description can be found in Ref. [53].

\subsection{Dynamical Consistency}

The unfolding of an attractor used in delay coordinate embedding [55] is the underlying idea of dynamical consistency. It can be determined using the premise behind the method of false nearest neighbors [56]. A linear subspace used for reduced order modeling is said to be dynamically consistent if the resultant trajectories are deterministic and smooth. The metric for dynamical consistency is defined as a ratio of the number of false nearest neighbors (FNN) over the total number of nearest neighbor pairs in a particular $k$-dimensional subspace:

$$
\zeta^{k}=1-\frac{N_{\mathrm{fnn}}^{k}}{N_{\mathrm{nn}}},
$$

where $N_{\text {fnn }}^{k}$ is the estimated number of FNNs in a $k$-dimensional subspace due to projection, and $N_{\text {nn }}$ is the total number of nearest neighbor pairs used in the estimation. If $\zeta^{k}$ is close to unity, then that $k$-dimensional subspace is dynamically consistent.

\section{$3.2 \quad$ Subspace Robustness}

Unlike LNM subspaces that are unique and not sensitive to changes in energy level, the robustness of the subspaces obtained by multivariate data analysis methods is not guaranteed. In order to 


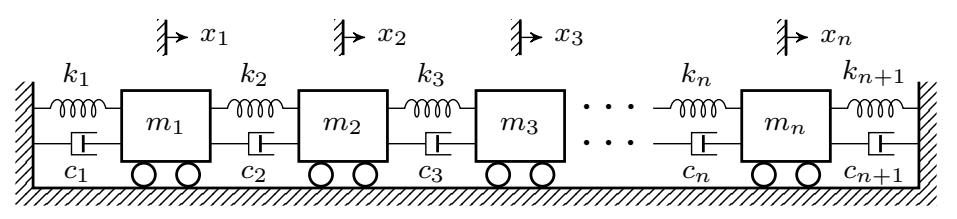

Figure 1: Schematic of the linear system

quantify the subspace robustness, the basis vectors which span the $k$-dimensional subspace for $s$ system realizations with different levels of energy are concatenated into a matrix $\mathbf{S} \in \mathbb{R}^{2 n \times k s}$. Then, the corresponding subspace robustness $\gamma_{s}^{k}$ is given by the following expression:

$$
\gamma_{s}^{k}=\left|1-\frac{4}{\pi} \arctan \sqrt{\frac{\sum_{i=k+1}^{2 n} \sigma_{i}^{2}}{\sum_{i=1}^{k} \sigma_{i}^{2}}}\right|,
$$

where $\sigma_{i}$ 's are proper orthogonal values of the matrix $\mathbf{S}$. If all the subspaces embedded in $\mathbf{S}$ are spanning the same subspace, then $\gamma=1$.

\section{Full-scale Models}

Complexity in dynamical systems can arise for different reasons. In nonlinear dynamical systems, it can be related to the size, nonlinearity, or a week coupling between the DOFs resulting in simultaneous presence of slow and fast dynamics. Here we study one large linear system as well as two nonlinear systems.

The linear system under investigation is an $n$-degree-of-freedom mass-spring-damper system, as shown in Fig. 1, where $n$ blocks of masses are connected in series to each other, as well as both sides of the support, by linear dampers and springs. The masses can vibrate in $x$-direction with no friction. The system is described by the following governing differential equations:

$$
\begin{cases}m_{i} \ddot{x}_{i}+\left(c_{i}+c_{i+1}\right) \dot{x}_{i}-c_{i+1} \dot{x}_{i+1}+ & \\ \left(k_{i}+k_{i+1}\right) x_{i}-k_{i+1} x_{i+1}=f_{i}(t), & \text { for } i=1 ; \\ m_{i} \ddot{x}_{i}-c_{i} \dot{x}_{i-1}+\left(c_{i}+c_{i+1}\right) \dot{x}_{i}-c_{i+1} \dot{x}_{i+1} & \\ -k_{i} x_{i-1}+\left(k_{i}+k_{i+1}\right) x_{i}-k_{i+1} x_{i+1}=f_{i}(t), & \text { for } 2 \leq i \leq n-1 ; \\ m_{i} \ddot{x}_{i}-c_{i} \dot{x}_{i-1}+\left(c_{i}+c_{i+1}\right) \dot{x}_{i}-k_{i} x_{i-1}+ & \\ \left(k_{i}+k_{i+1}\right) x_{i}=f_{i}(t), & \text { for } i=n,\end{cases}
$$

where $n \in \mathbb{N}$ is the number of the system's degrees of freedom and $f_{i}(t)$ is the external forcing applied to the $i$-th mass. Defining $\mathbf{z}=\left[\left\{x_{i}\right\}_{i=1}^{n},\left\{\dot{x}_{i}\right\}_{i=1}^{n}\right]^{T}$ as the vector of $2 n$ state variables, the full state-space model of the system can be obtained as follows:

$$
\dot{\mathbf{z}}=\left[\begin{array}{cc}
\mathbf{0} & \mathbf{I} \\
-\mathbf{M}^{-1} \mathbf{K} & -\mathbf{M}^{-1} \mathbf{C}
\end{array}\right] \mathbf{z}+\mathbf{f}_{\mathrm{e}}(t),
$$




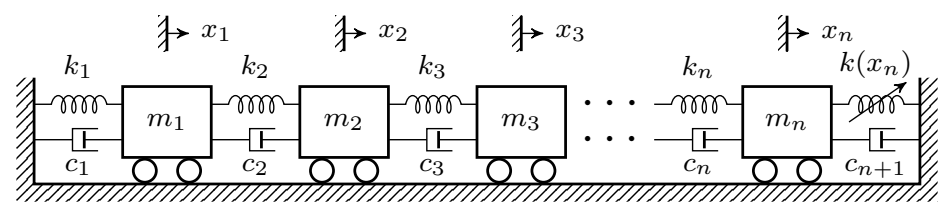

Figure 2: Schematic of the system with nonlinear spring coupling

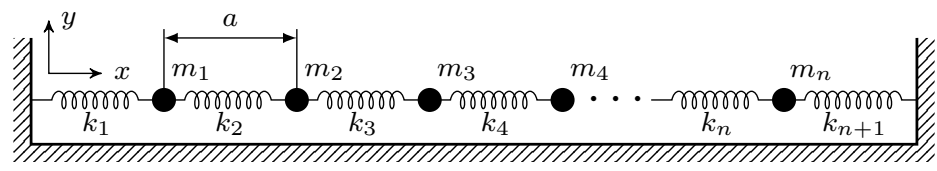

Figure 3: Schematic the mass-spring-grid system with geometric nonlinearity

where $\mathbf{f}_{\mathrm{e}}(t)=\left[[0, \ldots, 0]_{1 \times n},[1, \ldots, 1]_{1 \times n}\right]^{T} ; \mathbf{0} \in \mathbb{R}^{n \times n}$ is a zero matrix; $\mathbf{I} \in \mathbb{R}^{n \times n}$ is an identity matrix; and $\mathbf{M}, \mathbf{K}$, and $\mathbf{C}$ are $n \times n$ mass, stiffness, and damping matrices, respectively.

The first nonlinear system used here is obtained by adding a nonlinear spring to the linear system as shown in Fig. 2. In this case, the complexity is caused by the large size as well as the material nonlinearity. The system dynamics are described by the following full state-space equations of motion:

$$
\dot{\mathbf{z}}=\left[\begin{array}{cc}
\mathbf{0} & \mathbf{I} \\
-\mathbf{M}^{-1} \mathbf{K} & -\mathbf{M}^{-1} \mathbf{C}
\end{array}\right] \mathbf{z}+\mathbf{f}_{\mathrm{e}}+\mathbf{f}_{\text {nonlinear }}(\mathbf{z}),
$$

where everything is the same as for the linear model except nonlinear term $\mathbf{f}_{\text {nonlinear }}(\mathbf{z}) \in \mathbb{R}^{2 n}$, which has only one nonzero element: $\left[\mathbf{f}_{\text {nonlinear }}(\mathbf{z})\right]_{2 n}=-\alpha z_{n}^{3}$.

The third system will be called the mass-spring-grid system throughout this paper. It has the same design and arrangement as the first system, but with a pretension in the springs. Each spring is assumed to have a corresponding damper acting in parallel. The system is allowed to vibrate in both $x$ and $y$ directions as a grid of equidistant masses, dampers, and springs shown in Fig. 3. In case the system is forced only in the $x$-direction, Eq. (8) is sufficient to describe it. However, any small deviation from $x$-directional oscillation will cause geometric nonlinearity in the motion. For the purpose of this paper, we only excite this system in the $y$-direction. The governing differential equations for each $i$-th mass are given in Appendix A.

The state-space vector to model this system is a vector of $4 n$ variables defined as $\mathbf{z}=\left[\left\{y_{i}\right\}_{i=1}^{n}\right.$, $\left.\left\{x_{i}\right\}_{i=1}^{n},\left\{\dot{y}_{i}\right\}_{i=1}^{n},\left\{\dot{x}_{i}\right\}_{i=1}^{n}\right]^{T} \in \mathbb{R}^{4 n}$. Thus, Eq. (15) and Eq. (18) from the appendix can be rewritten as follows:

$$
\dot{\mathbf{z}}=\left[\begin{array}{cccc}
\mathbf{0} & \mathbf{0} & \mathbf{I} & \mathbf{0} \\
\mathbf{0} & \mathbf{0} & \mathbf{0} & \mathbf{I} \\
-\mathbf{M}^{-1} \mathbf{K} & \mathbf{0} & -\mathbf{M}^{-1} \mathbf{C} & \mathbf{0} \\
\mathbf{0} & -\mathbf{M}^{-1} \mathbf{K} & \mathbf{0} & -\mathbf{M}^{-1} \mathbf{C}
\end{array}\right] \mathbf{z}+\left[\begin{array}{c}
\mathbf{0}_{n \times 1} \\
\mathbf{0}_{n \times 1} \\
\mathbf{f}_{n, y} \\
\mathbf{f}_{n, x}
\end{array}\right]+\mathbf{f}_{\mathrm{e}}
$$

where $\mathbf{f}_{n, y}, \mathbf{f}_{n, x}$, and $\mathbf{f}_{\mathrm{e}}$ are given in Appendix A; and $\mathbf{0}, \mathbf{I}, \mathbf{M}, \mathbf{K}$, and $\mathbf{C}$ are $n \times n$ zero, identity, mass, stiffness, and damping matrices, respectively.

\section{$5 \quad$ Results and Discussion}

The MOR objective is to develop persistent ROMs for harmonically excited systems considered in this paper. These systems will be excited by a force with frequency close to the first natural frequency 
of the corresponding linear(ized) system. Modal subspaces for model reduction can be obtained from different types of excitations, including both harmonic and random. With random forcing, we are more likely to explore nearly all the state-space of the system and excite all dominant frequencies. However, the particular forcing function has to be carefully selected, especially for the systems that have combined slow and fast dynamics. This is to limit the contamination of the identified modes by forcing that can obscure the true modal structure of the system.

For the first and the second system, white noise is used to excite the system because there are no relatively fast dynamics in the presence of slow dynamics. For the third system, we use colored white noise with the cut-off frequency of $6 \mathrm{~Hz}$, which, for our numerical example, will be around the frequency of the linearized system's ninth LNM. This allows the excitation of lower modal frequencies, while limiting contamination from high frequency modes that do not get excited in practice. Also, the external excitation containing a range of frequencies ensures that the geometrical nonlinearity caused by the $x$-direction oscillations is observable while these oscillations are not contaminated by noise.

We did 12 independent simulations for each system subjected to external stochastic excitations. The obtained time series from each simulation had different levels of energy imposed by changing the amplitude of forcing. For fair comparison purposes, we need to be consistent with the selection of the total simulation time for each system. Thus, each simulation was done for a total time equal to 100 cycles of a harmonic forcing, with the frequency equal to $110 \%$ of the first natural frequency of the corresponding linearized system. With the chosen parameters for the systems, the total simulation times were equal to $709.8 \mathrm{sec}$ for the linear, $495.1 \mathrm{sec}$ for the nonlinear spring, and $120.8 \mathrm{sec}$ for the mass-spring-grid systems. We recorded 100 data samples in each cycle of applied external forcing. Therefore, a total of 10,000 data points were recorded from each simulation.

In each case, POD and SOD were used to extract the modes out of each data set. The first $k$ dominant modes identified from each simulation independently, spanning $12 k$-dimensional subspaces, were concatenated into the matrix $\mathbf{S}$ as explained in Section 3.2. Singular value decomposition was applied to matrix $\mathbf{S}$ in order to extract the singular modes and the corresponding singular values. Using singular values and Eq. (7), the robustness of the $k$-dimensional subspaces were evaluated for each model and decomposition scheme.

The corresponding singular modes were used to obtain projections of the full-scale models' harmonically excited trajectories onto them. Using the procedure outlined in Section 3.1, the dynamical consistency of the resulting trajectories for all the $k$-dimensional subspaces in the full $n$-dimensional vector space were obtained. Please note that no matter how we obtain the subspace for model reduction, the calculation of the dynamical consistency is meaningful only for the deterministic trajectories. The dynamical consistencies were obtained for five deterministic trajectories, each corresponding to different forcing amplitudes, and then averaged out. In case both subspace robustness and dynamical consistencies of the extracted modes were close to unity, we considered them as suitable for persistent MOR.

The parameters of the linear system were fixed as follows:

$$
n=100, m=1 \mathrm{~kg}, k=1000 \mathrm{~N} / \mathrm{M}, c=0.048 \mathrm{~N} \cdot \mathrm{M} / \mathrm{s} .
$$

The obtained POMs and SOMs for this system are used to get the subspace robustness and dynamical consistency of the ROM subspaces. For the randomly driven linear system, as depicted in Fig. 4, the SOD subspace robustness metric reaches and stays close to unity for $k \geq 3$. POD subspace robustness is close to unity at $k=2$ and $k=3$. It drops at $k=4$ and again reaches unity at $k=25$ 

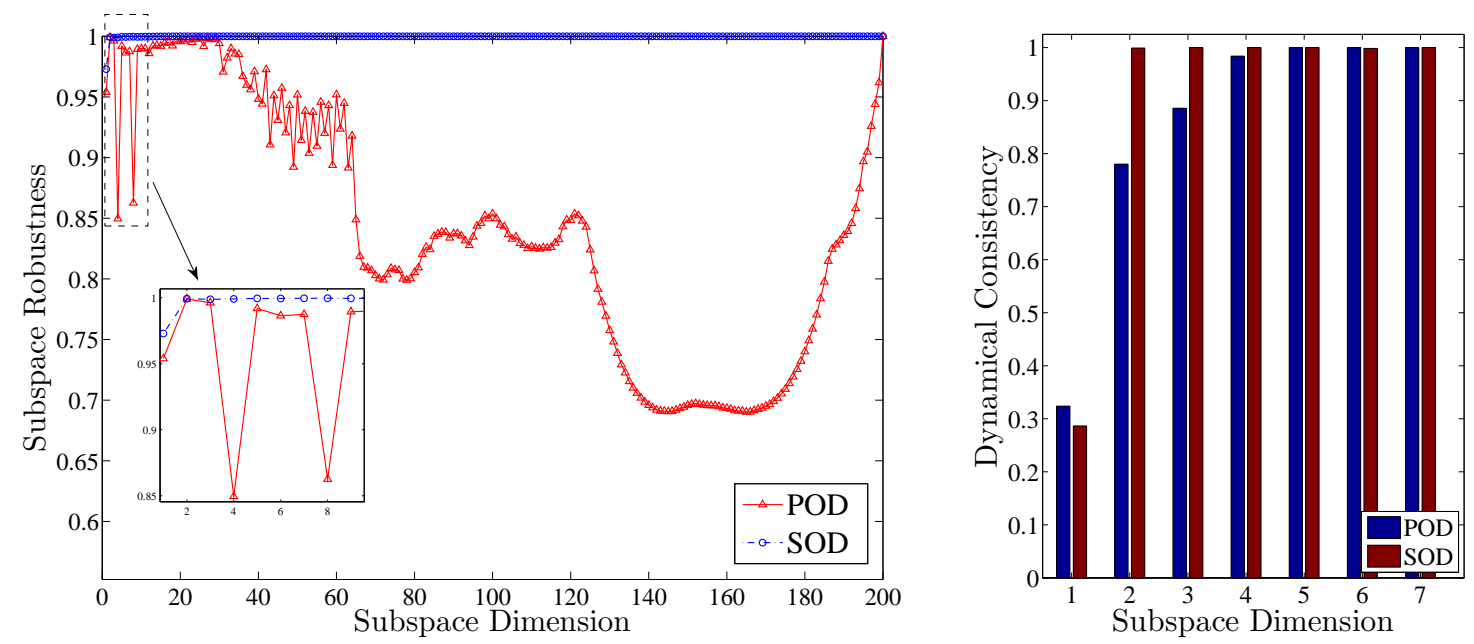

Figure 4: Subspace robustness (left) and dynamical consistency (right) for randomly driven linear system

in a non-monotonic manner.

The POD subspace robustness does not behave monotonically and does not stay close to unity once it reaches it. Therefore, in the cases considered, POMs cannot approximate fixed LNMs in a robust manner, except maybe a few lower modes. This shows that POMs are not robust under the limited time-history constraints of high-dimensional data, which makes them unreliable for persistent MOR. In contrast, the SOD subspace robustness monotonically increases, reaches unity for a low dimension, and does not fluctuate thereafter.

Figure 4 also shows the dynamical consistency of POD- and SOD-based subspaces for the randomly driven linear system. For both POD and SOD, the dynamical consistencies are similar reaching unity at $k=2$ for SOD and $k=5$ for POD. This means that the projection of the linear system's deterministic trajectories onto the five-dimensional POD-based, or the two-dimensional SOD-based, dominant subspaces has no singular point or intersection with itself-or, they do not violate the uniqueness of the deterministic evolution.

The linear system subjected to harmonic excitation was simulated using the POD- and SODbased ROMs via Eq. (2). The phase portraits for the vibrations of the thirtieth mass are depicted in Fig. 5 and Fig. 6. The ROM simulations results show a very good visual correspondence to the full-scale system using both POD and SOD. Both methods are able to capture the dynamics in two- and three-dimensional ROMs and none of them outperformed the other irrespective of the robustness of the corresponding subspaces.

A question arises as to why some relatively non-robust POD subspace-based ROMs, like the fourdimensional model, still correlate with the full-scale model. It should be noted that the subspace robustness metric is of more importance for lower dimensions, since they possess most of the energy of the system. The two- and three-dimensional POD subspaces for the linear system are robust and capture most of the system's energy, thus providing for good ROMs of the system. Increasing the dimension of the ROM reduces the robustness of the associated POD subspace to 0.85 but it does not affect its accuracy or stability. This is mainly due to the fact that the fourth POM does not capture enough associated energy to have a sizable effect on the corresponding ROM. This also explains why the robustness of MOR based on POD has not been of much research concern for linear systems. As shown in Fig. 7, POD captures most of the energy in the very first few modes, which are robust to the changes in the energy of the system. Therefore, any suitably developed POD-based 

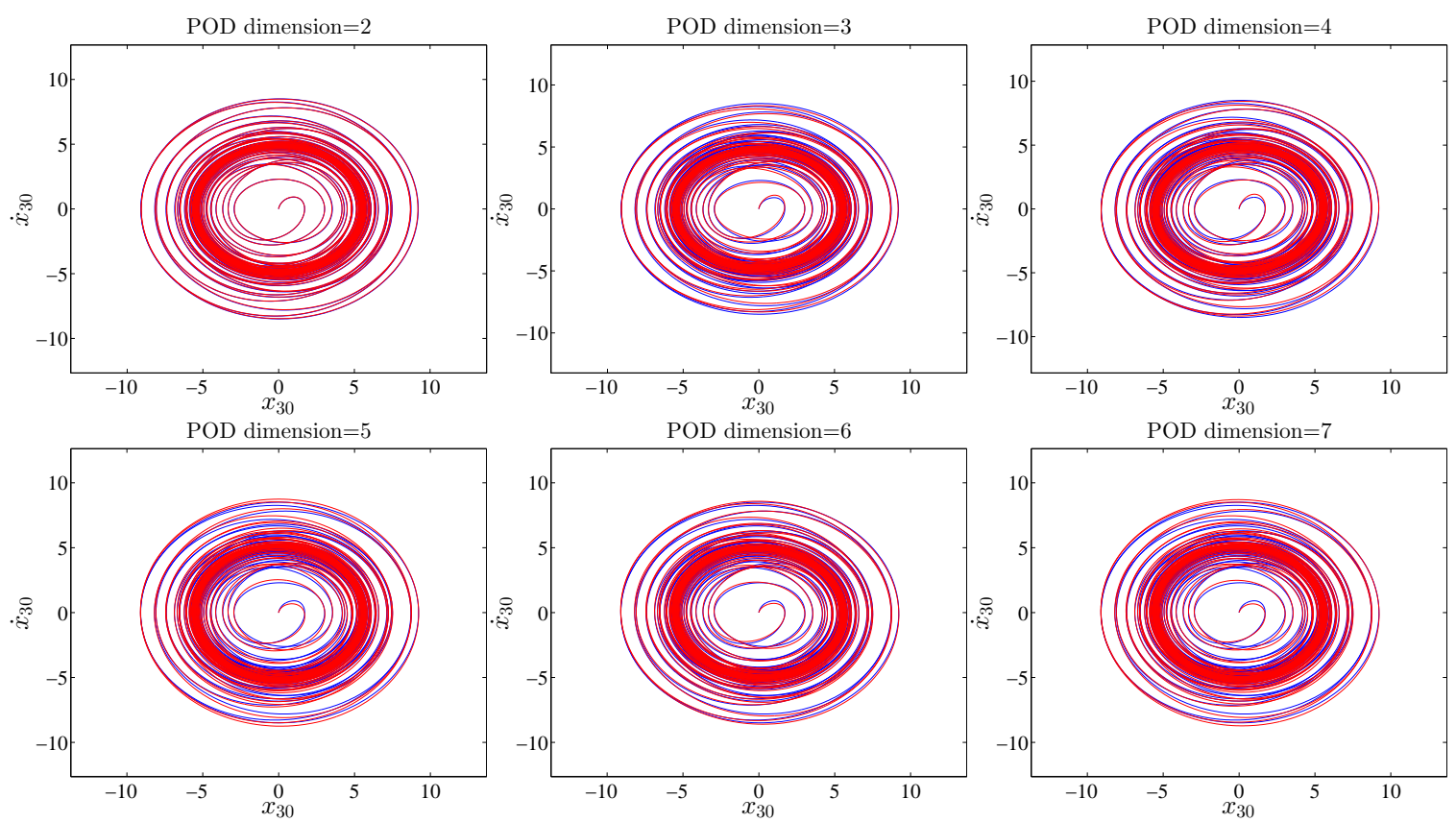

Figure 5: POD-based ROMs of the linear system for $\Omega=5.52 \mathrm{~Hz}$ and $q_{0}=1$

ROM could probably account for other similar conditions.

ROMs for nonlinear systems are expected to be more sensitive to the robustness of the corresponding MOR linear subspaces. Therefore, in case the low-dimensional subspaces have good robustness, the non-robust higher dimensional subspace may destabilize the numerical scheme for the model, or at least adversely affect its accuracy.

For investigation of the system with nonlinear spring coupling, the number of DOFs was set to 60 and the other parameters were fixed as follows:

$$
m=1 \mathrm{~kg}, k=3600 \mathrm{~N} / \mathrm{M}, c=720 \mathrm{~N} . \mathrm{M} / \mathrm{s}, \alpha=2 \text {. }
$$

This results in a rich dynamic response with two stable and one unstable static equilibrium points. Subjected to harmonic forcing with $\Omega=9.97 \mathrm{~Hz}$, and using the forcing amplitude as a bifurcation parameter, the corresponding bifurcation diagram is plotted using the full scale model of the system as shown in Fig. 8. Our particular aim for the persistent ROM is to reproduce these bifurcation results, which will demonstrate robustness of ROM over a range of forcing amplitudes or different input energy levels.

The subspace robustness and dynamical consistency for this system are depicted in Fig. 9. The robustness for the SOD subspaces reaches unity at $k=4$, while for POD it does not happen until the very end. POD subspace robustness is fluctuating and sometimes getting worse as the subspace dimension increases. These fluctuations are of greater importance for lower dimensional subspaces since most of the system's response energy is captured in these subspaces. The dynamical consistency for both methods is similar and reaches unity at $k=2$. At $k=5$, however, the dynamical consistency of the SOD method slightly drops, which may affect the accuracy of the corresponding ROM.

While two- and three-dimensional POD subspace robustness are relatively close to unity, they do not account for a significant portion of the system's total energy to provide stable ROMs. The robustness of the four-dimensional POD subspace is low, which causes the diverging results of the 

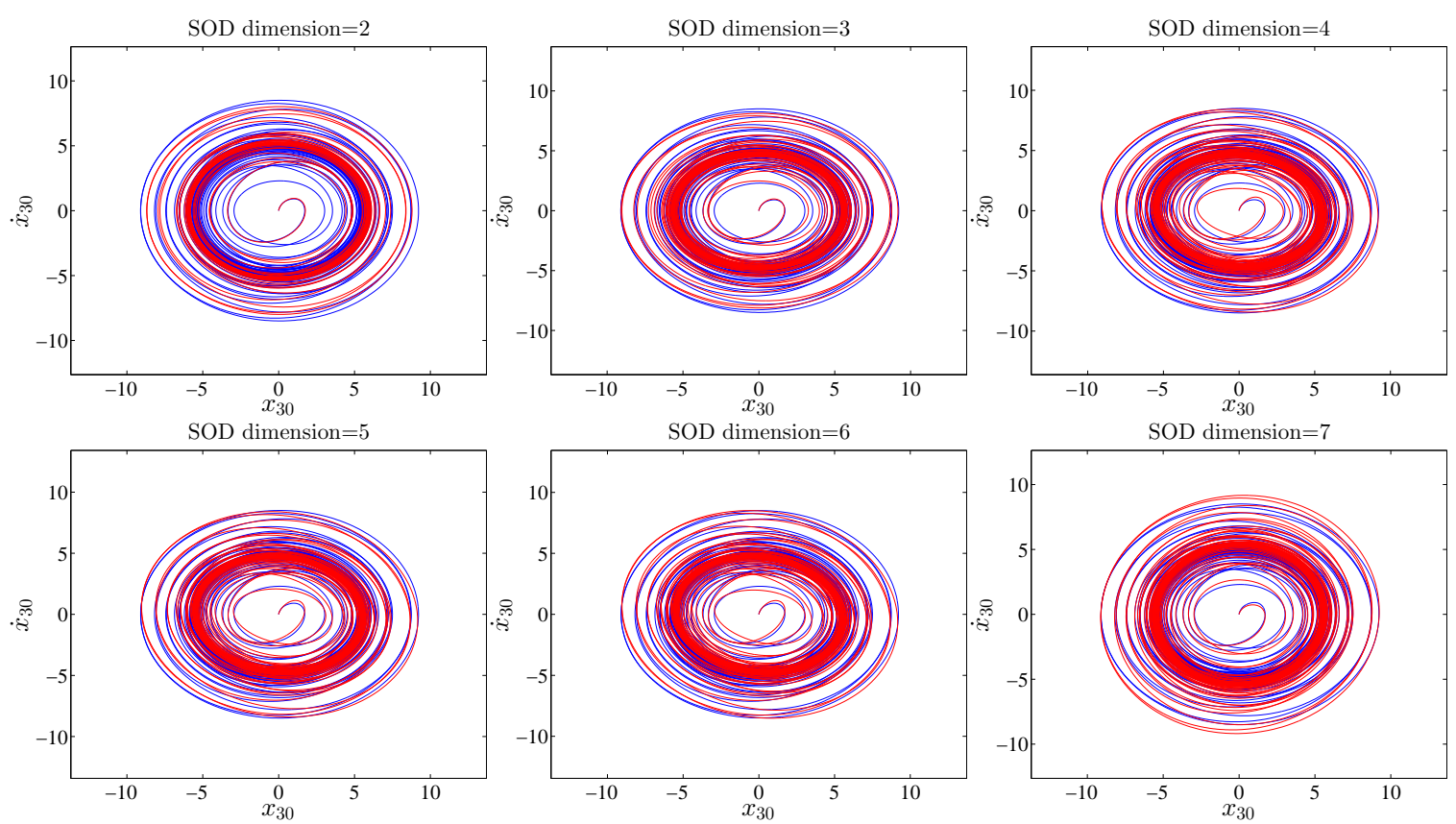

Figure 6: SOD-based ROMs of the linear system for $\Omega=5.52 \mathrm{~Hz}$ and $q_{0}=1$

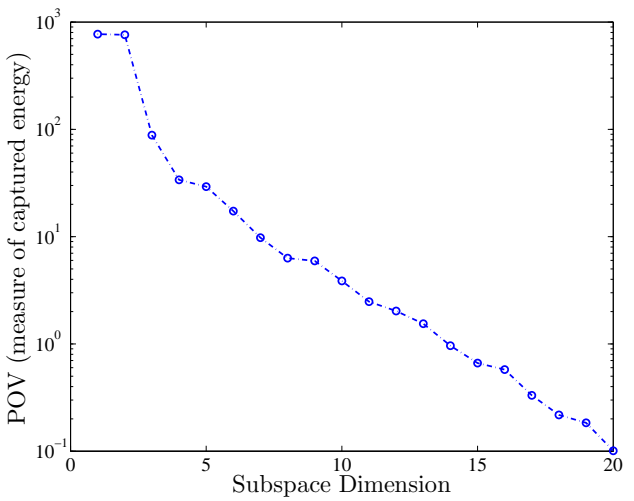

Figure 7: Captured energy vesus number of the modes for the linear system

corresponding ROM simulation. The five-dimensional POD subspace has better robustness, and the simulations showed that it provides stable ROM, yet is not robust enough to accurately reproduce the bifurcation diagram. Since the six-dimensional POD-based ROM has better robustness and captures more energy, it results in stable and accurate simulations.

One- through three-dimensional SOD subspaces do not result in persistent ROMs because their subspace robustness is relatively low and also they do not capture enough energy of the system. Four- and higher-dimensional SOD subspaces are robust and provide persistent ROMs capable of reproducing the bifurcation diagram of the full-scale system.

The lowest dimensional ROM which provides accurate and robust results is four-dimensional for SOD and six-dimensional for POD. The corresponding bifurcation diagrams are shown in Fig. 10. These ROMs are more than fifty times faster in simulation than the full scale model. Thus, we used a finer increment size for the forcing amplitude to provide more details in the bifurcation diagrams of the system. Comparing these diagrams with the reference diagram shown in Fig. 8, there is a 


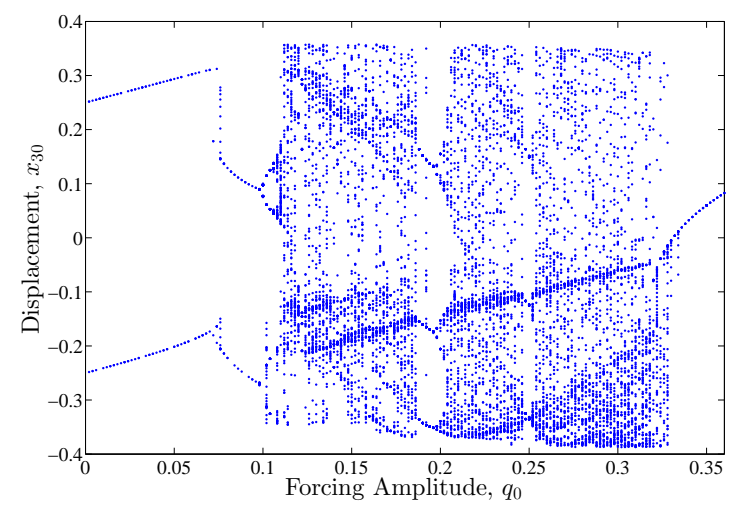

Figure 8: Bifurcation diagram for full scale nonlinear system for harmonic forcing with $\omega=9.97 \mathrm{~Hz}$
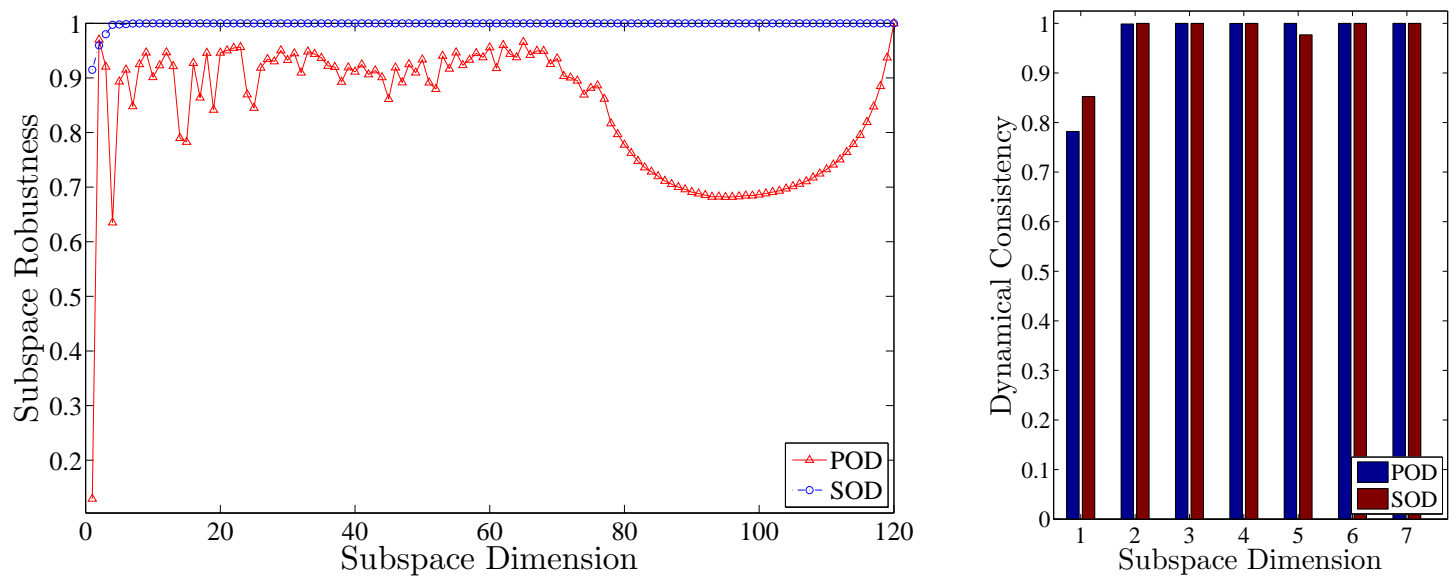

Figure 9: Subspace robustness (left) and dynamical consistency (right) for randomly driven nonlinear system

close match between those of the six-dimensional POD and the full scale model. For the SOD, the bifurcation diagram is little shrunk around $q_{0}=0.33$. While this bifurcation diagram is not strictly accurate, it still provides a faithful qualitative description the full-scale system's dynamics. In addition, the results for the six-dimensional SOD are as good as the six-dimensional POD, while the test shows that the four-dimensional POD is not even stable, due to the significant drop of its subspace robustness at $k=4$.

In Fig. 11, six-dimensional POD and four-dimensional SOD ROMs are compared to the full-scale model driven by the harmonic forcing with $\Omega=9.97 \mathrm{~Hz}$ and amplitudes of $0.05,0.14,0.28$, and 0.35 . The four-dimensional SOD model successfully competes with the six-dimensional POD model. For smaller amplitudes, four-dimensional SOD even outperforms the six-dimensional POD. In addition, Fig. 12 shows how the relative accuracy of the SOD-based ROMs drops for $k=5$ as compared to $k=4$ and 6 . This can be explained by the drop in the dynamical consistency of SOD for $k=5$, which was shown in Fig. 9.

For the mass-spring-grid system consisting of twenty masses, specifying the following parameters will result in a rich dynamical behavior:

$$
n=20, m=1 \mathrm{~kg}, k=1000 \mathrm{~N} / \mathrm{M}, c=4.23 \mathrm{~N} . \mathrm{M} / \mathrm{s}, a=1.01 \mathrm{~m}, l=1 \mathrm{~m} .
$$

The subspace robustness for POD-based MOR of this system is close to unity for $k=1, \ldots, 4$ and 

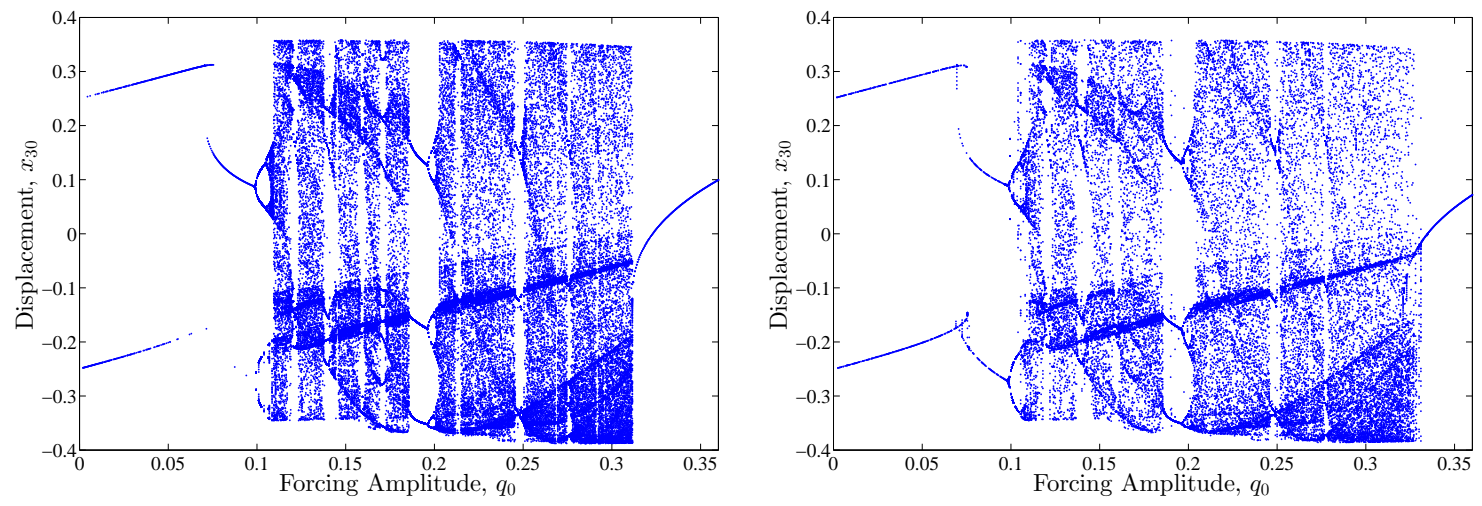

Figure 10: Bifurcation diagram for ROMs of the nonlinear system for harmonic forcing with $\omega=9.97 \mathrm{~Hz}:$ fourdimensional SOD (left); six-dimensional POD (right)
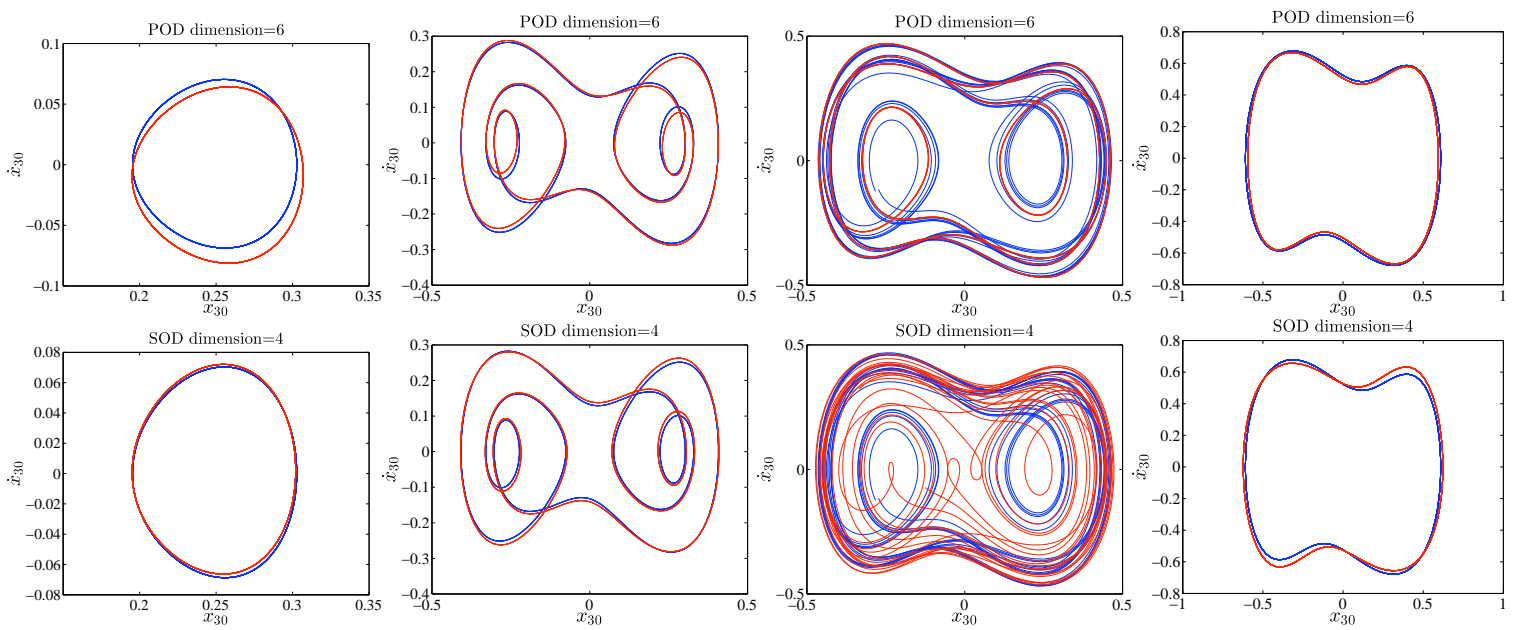

Figure 11: Phase portraits of the 30-th mass of the nonlinear system obtained from full scale (blue) and reduced order models (red): four-dimensional POD-based ROMs (top); six-dimensional SOD-based ROMs (bottom)

drops to 0.6 at $k=5$, as shown in Fig. 13. For SOD, subspace robustness starts at near 0.85 for $k=1$, monotonically increases with the increase in the dimension, and saturates at 1 near $k=20$. Also, three- and higher-dimensional POD-based ROMs are dynamically consistent, while for SOD, five- and higher-dimensional subspaces are dynamically consistent. The importance of subspace robustness and dynamical consistency metrics for identifying the optimal MOR subspace is reflected in Fig. 14, where POD-based ROMs lose their stability as subspace robustness drops for $k=5$. While the five-dimensional ROM is still stable, for the $k=6,7$, or 8 , it loses its stability. The importance of monotonically increasing subspace robustness for SOD-based ROMs is illustrated in Fig. 15. These ROMs become and remain stable as the robustness metric approaches unity and stays there.

Therefore, for all the three types of systems under investigation, the subspace robustness and dynamical consistency of the ROMs were good indicators for the stability, accuracy, and reliability of the corresponding ROMs. Not only does any deviation of these metrics from unity reduce the accuracy, but it may also destabilize the corresponding ROMs. In particular, in contrast to POD, when SOD-based ROMs become stable, they stay stable for higher dimensional reductions. This is directly correlated with monotonic improvement in the SOD subspace robustness, which also 

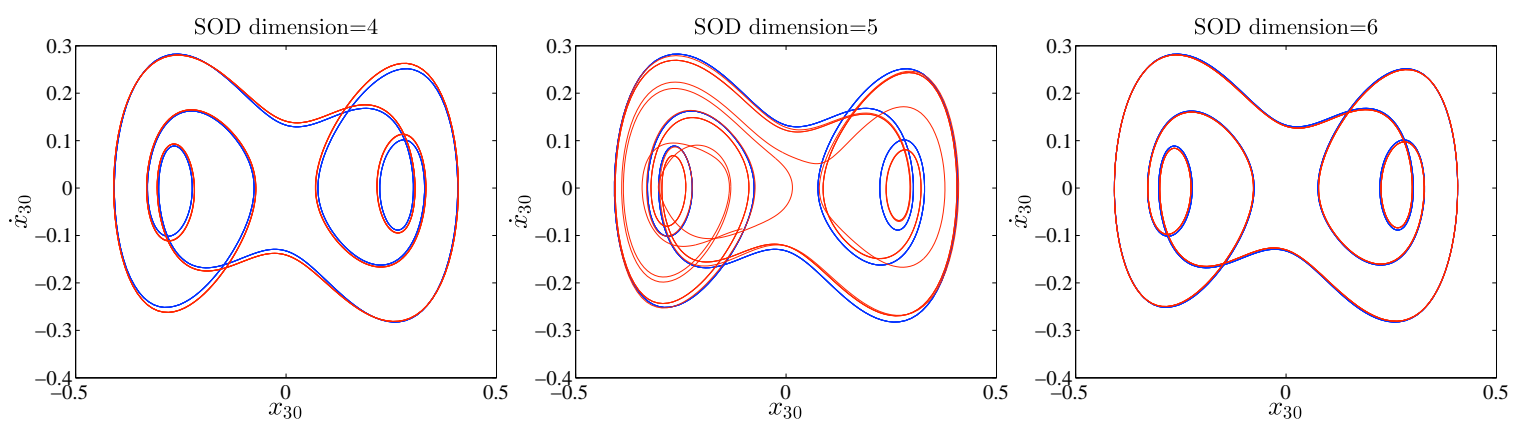

Figure 12: Phase portraits of the 30-th mass of the nonlinear system obtained from full scale (blue) and SOD-based ROMS (red)
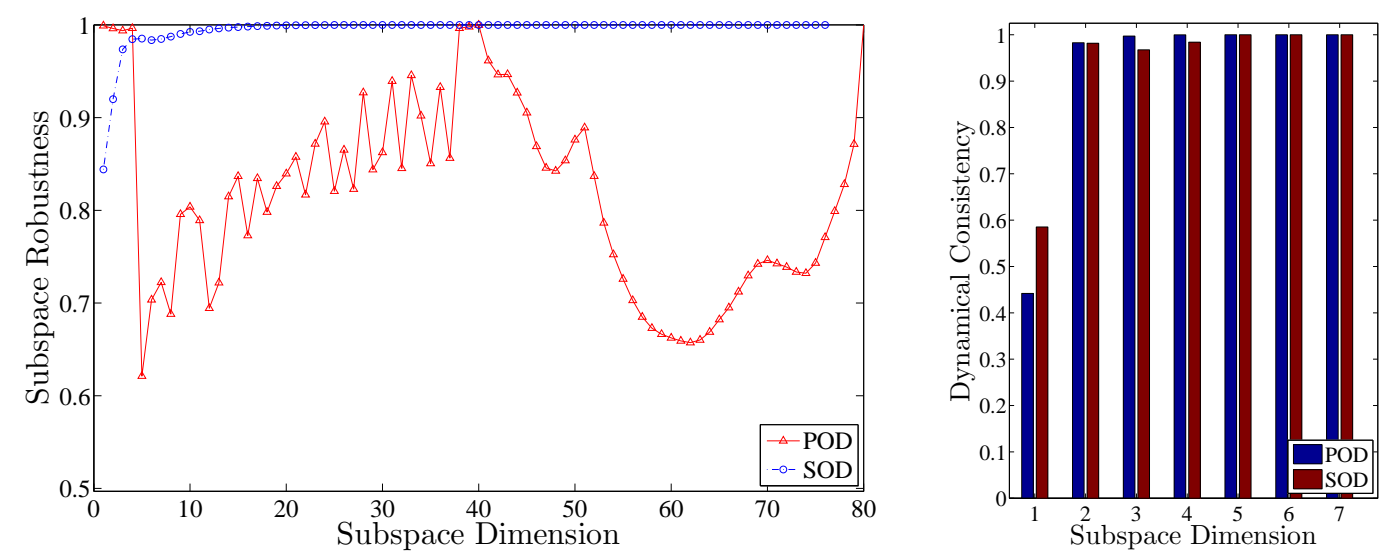

Figure 13: Subspace robustness (left) and averaged dynamical consistency (right) for randomly driven nonlinear mass-grid system

correlates with the improved accuracy of the corresponding ROMs. For the POD subspaces, we do not observe this monotonic increase in the robustness, which may drop precipitously as the subspace dimension increases. This may cause the loss of stability in the corresponding ROM for the nonlinear dynamical system.

The performance of the studied framework for identifying the optimal subspaces for persistent MOR was verified by reproducing full scale model simulations for a range of amplitudes and frequencies of external excitation, and initial conditions. For the second and the third system, where more complexities were introduced through material and geometrical nonlinearities, the SOD-based model outperformed the POD-based models by providing more consistent and persistent reductions. Also, we should emphasize that the obtained fast, stable, and robust-over-a-wide-energy-range ROMs enabled us to study these systems in more detail.

\section{Conclusions}

A persistent MOR for dynamical systems was investigated for one example of a large linear system and two examples of large and complex nonlinear systems. A framework based on subspace robustness and dynamical consistency was shown to be successful in identifying the robust subspaces for the development of persistent ROMs. To verify the performance of the framework, the simulation results of the full scale models were reproduced using the ROMs. In particular, SOD-based ROMs 

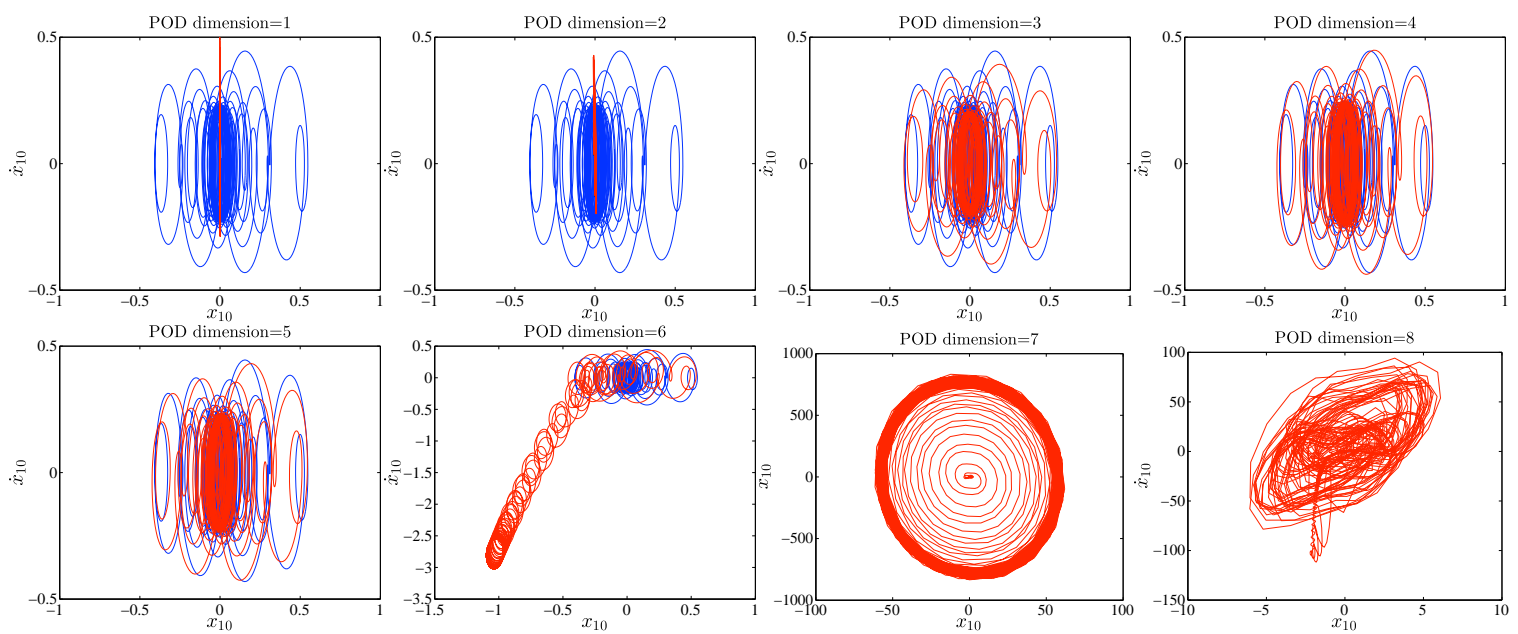

Figure 14: Phase portraits of the 10-th mass of the nonlinear mass-grid system obtained from full scale model (blue) for $\Omega=28.2 \mathrm{~Hz}$ and $q_{0}=1$ compared to 1- through 8-dimensional POD-based ROMs (red)
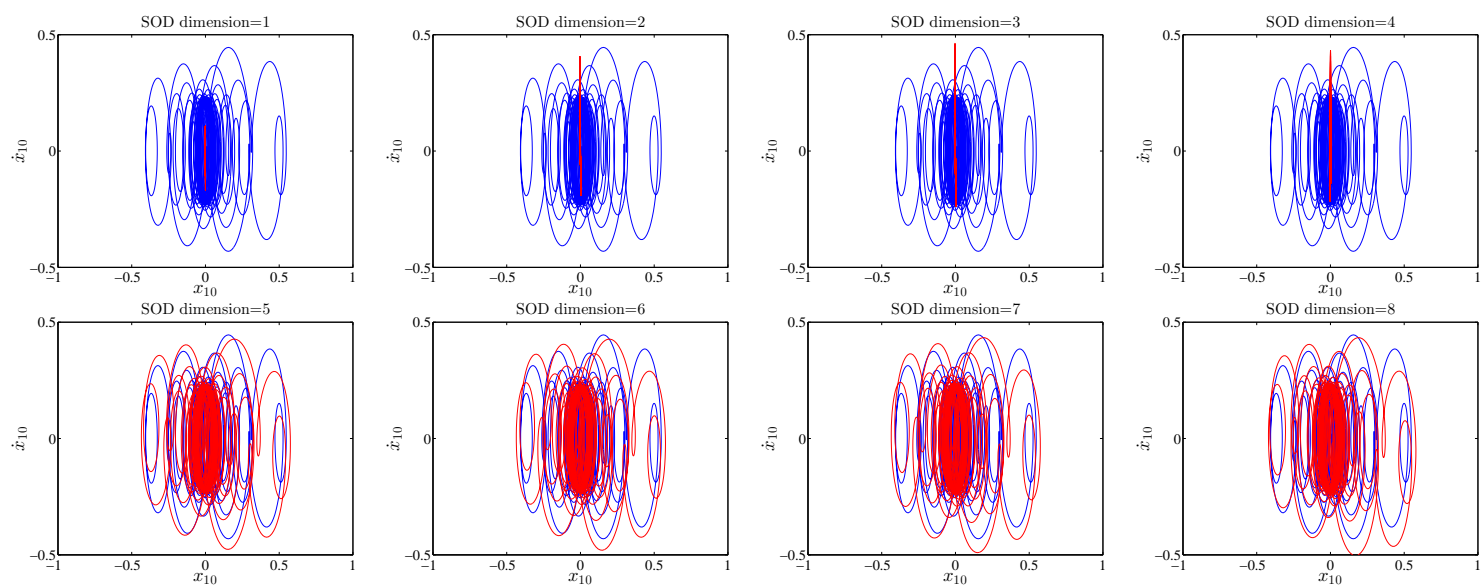

Figure 15: Phase portraits of the 10-th mass of the nonlinear mass-grid system obtained from full scale model (blue) for $\Omega=28.2 \mathrm{~Hz}$ and $q_{0}=1$ compared to 1- through 8-dimensional SOD-based ROMs (red)

outperformed the POD-based ones in terms of the stability and robustness of the model. Also, the obtained persistent ROMs could be successfully used to study the dynamics of computationally expensive complex models for a relatively wide range of parameters and conditions.

The persistent MOR framework presented in this paper can be used for improving all the projection-based methods and has a good performance for parametric studies within their domain of interest. However, the parametric study is only limited to the defined range of parameters and the persistent model is expected to be valid only in this range. In our study, we focused on a relatively wide range of parameters where the complex systems exhibit interesting dynamics, which includes linearity, nonlinearity, periodicity, intermittence, and chaos. In future work, one needs to study the validity of persistent ROMs outside the domain of interest. Future efforts may focus on increasing the size of the domain for persistent MOR. 


\section{Acknowledgement}

This paper is based upon work supported by the National Science Foundation under Grant No. 1100031.

\section{References}

[1] Benner, P., Mehrmann, V., and Sorensen, D. C., 2005. Dimension reduction of large-scale systems, vol. 45. Springer.

[2] Ilbeigi, S., and Chelidze, D., 2016. "Model order reduction of nonlinear euler-bernoulli beam". In Nonlinear Dynamics, Volume 1. Springer, pp. 377-385.

[3] Antoulas, A. C., Ionutiu, R., Martins, N., ter Maten, E. J. W., Mohaghegh, K., Pulch, R., Rommes, J., Saadvandi, M., and Striebel, M., 2015. "Model order reductionmethods, concepts and properties".

[4] Maier, D., Hager, C., Hetzler, H., Fillot, N., Vergne, P., Dureisseix, D., and Seemann, W., 2015. "A nonlinear model order reduction approach to the elastohydrodynamic problem". Tribology International, $\mathbf{8 2}$, pp. 484-492.

[5] Kudryavtsev, M., Rudnyi, E., Korvink, J., Hohlfeld, D., and Bechtold, T., 2015. "Computationally efficient and stable order reduction methods for a large-scale model of mems piezoelectric energy harvester". Microelectronics Reliability, 55 (5) , pp. 747-757.

[6] Benner, P., and Feng, L. "Model order reduction for coupled problems".

[7] Balajewicz, M., Amsallem, D., and Farhat, C., 2015. "Projection-based model reduction for contact problems". arXiv preprint arXiv:1503.01000 .

[8] Rathinam, M., and Petzold, L. R., 2003. "A new look at proper orthogonal decomposition". SIAM Journal on Numerical Analysis, 41 (5), pp. 1893-1925.

[9] Willcox, K., and Peraire, J., 2002. "Balanced model reduction via the proper orthogonal decomposition". AIAA journal, 40 (11), pp. 2323-2330.

[10] Benner, P., and Breiten, T., 2015. "Two-sided projection methods for nonlinear model order reduction". SIAM Journal on Scientific Computing, 37 (2), pp. B239-B260.

[11] Georgiou, I., 2005. "Advanced proper orthogonal decomposition tools: using reduced order models to identify normal modes of vibration and slow invariant manifolds in the dynamics of planar nonlinear rods". Nonlinear dynamics, 41 (1-3), pp. 69-110.

[12] Ghasemi, M., Yang, Y., Gildin, E., Efendiev, Y., Calo, V., et al., 2015. "Fast multiscale reservoir simulations using pod-deim model reduction". In SPE Reservoir Simulation Symposium, Society of Petroleum Engineers.

[13] Kerschen, G., Golinval, J.-c., Vakakis, A. F., and Bergman, L. A., 2005. "The method of proper orthogonal decomposition for dynamical characterization and order reduction of mechanical systems: an overview". Nonlinear dynamics, 41 (1-3), pp. 147-169.

[14] Foias, C., Jolly, M., Kevrekidis, I., Sell, G., and Titi, E., 1988. "On the computation of inertial manifolds". Physics Letters A, 131 (7), pp. 433-436. 
[15] Pesheck, E., Pierre, C., and Shaw, S., 2002. "A new galerkin-based approach for accurate nonlinear normal modes through invariant manifolds". Journal of sound and vibration, 249 (5), pp. 971-993.

[16] Feldmann, P., and Freund, R. W., 1995. "Efficient linear circuit analysis by padé approximation via the lanczos process". Computer-Aided Design of Integrated Circuits and Systems, IEEE Transactions on, 14 (5), pp. 639-649.

[17] Glover, K., 1984. "All optimal hankel-norm approximations of linear multivariable systems and their 1,-error bounds". International journal of control, 39 (6), pp. 1115-1193.

[18] Zhou, H., Su, X., Song, Y.-D., and Yan, Q., 2015. "Hankel-norm model reduction for delayed fuzzy systems". In Control and Decision Conference (CCDC), 2015 27th Chinese, IEEE, pp. 964-968.

[19] Phillips, J. R., Daniel, L., and Silveira, L. M., 2003. "Guaranteed passive balancing transformations for model order reduction". Computer-Aided Design of Integrated Circuits and Systems, IEEE Transactions on, 22 (8), pp. 1027-1041.

[20] Baur, U., Benner, P., and Feng, L., 2014. "Model order reduction for linear and nonlinear systems: a system-theoretic perspective". Archives of Computational Methods in Engineering, 21 (4), pp. 331-358.

[21] Bai, Z., 2002. "Krylov subspace techniques for reduced-order modeling of large-scale dynamical systems". Applied numerical mathematics, 43 (1-2), pp. 9-44.

[22] Phillips, J. R., 2003. "Projection-based approaches for model reduction of weakly nonlinear, time-varying systems". IEEE Transactions on computer-aided design of integrated circuits and systems, 22 (2), pp. 171-187.

[23] Broomhead, D., and Kirby, M., 2005. "Dimensionality reduction using secant-based projection methods: The induced dynamics in projected systems". Nonlinear Dynamics, 41 (1) , pp. 47-67.

[24] GlÖsmann, P., and Kreuzer, E., 2005. "Nonlinear system analysis with karhunen-loève transform”. Nonlinear Dynamics, 41 (1) , pp. 111-128.

[25] Smith, T. R., Moehlis, J., and Holmes, P., 2005. "Low-dimensional modelling of turbulence using the proper orthogonal decomposition: a tutorial". Nonlinear Dynamics, 41 (1), pp. 275307.

[26] Lall, S., Marsden, J. E., and Glavaški, S., 1999. "Empirical model reduction of controlled nonlinear systems". International Federation of Automatic Control.

[27] Lall, S., Marsden, J. E., and Glavaški, S., 2002. "A subspace approach to balanced truncation for model reduction of nonlinear control systems". International journal of robust and nonlinear control, 12 (6) , pp. 519-535.

[28] Condon, M., and Ivanov, R., 2004. "Empirical balanced truncation of nonlinear systems". Journal of Nonlinear Science, 14 (5), pp. 405-414. 
[29] Marques, F. D., and Anderson, J., 2001. "Identification and prediction of unsteady transonic aerodynamic loads by multi-layer functionals". Journal of Fluids and Structures, 15 (1), pp. 83106.

[30] Lucia, D. J., Beran, P. S., and Silva, W. A., 2004. "Reduced-order modeling: new approaches for computational physics". Progress in Aerospace Sciences, 40 (1), pp. 51-117.

[31] Brunovsky, P., 1993. "Theory of invariant manifolds and its applications to differential equations". UTMS 93, 41, pp. 93-41.

[32] Nouy, A., 2010. "A priori model reduction through proper generalized decomposition for solving time-dependent partial differential equations". Computer Methods in Applied Mechanics and Engineering, 199 (23) , pp. 1603-1626.

[33] Chevreuil, M., and Nouy, A., 2012. "Model order reduction based on proper generalized decomposition for the propagation of uncertainties in structural dynamics". International Journal for Numerical Methods in Engineering, 89 (2), pp. 241-268.

[34] Chinesta, F., Ammar, A., and Cueto, E., 2010. "Recent advances and new challenges in the use of the proper generalized decomposition for solving multidimensional models". Archives of Computational methods in Engineering, 17 (4), pp. 327-350.

[35] Pruliere, E., Chinesta, F., and Ammar, A., 2010. "On the deterministic solution of multidimensional parametric models using the proper generalized decomposition". Mathematics and Computers in Simulation, 81 (4), pp. 791-810.

[36] Ladevèze, P., and Chamoin, L., 2011. "On the verification of model reduction methods based on the proper generalized decomposition". Computer Methods in Applied Mechanics and Engineering, 200 (23) , pp. 2032-2047.

[37] Chinesta, F., Ladeveze, P., and Cueto, E., 2011. "A short review on model order reduction based on proper generalized decomposition". Archives of Computational Methods in Engineering, 18 (4), p. 395.

[38] Shaw, S., and Pierre, C., 1991. "Non-linear normal modes and invariant manifolds". Journal of sound and Vibration, 150 (1), pp. 170-173.

[39] Pesheck, E., Pierre, C., and Shaw, S. W., 2002. "Modal reduction of a nonlinear rotating beam through nonlinear normal modes*”. Journal of vibration and Acoustics, 124 (2), pp. 229-236.

[40] Kerschen, G., Peeters, M., Golinval, J.-C., and Vakakis, A. F., 2009. "Nonlinear normal modes, part i: A useful framework for the structural dynamicist". Mechanical Systems and Signal Processing, 23 (1), pp. 170-194.

[41] Grolet, A., and Thouverez, F., 2015. "Computing multiple periodic solutions of nonlinear vibration problems using the harmonic balance method and groebner bases". Mechanical Systems and Signal Processing, 52, pp. 529-547.

[42] Mohammadali, M., and Ahmadian, H., 2014. "Efficient model order reduction of structural dynamic systems with local nonlinearities under periodic motion". Shock and Vibration, 2014. 
[43] Blanc, F., Touzé, C., Mercier, J.-F., Ege, K., and Ben-Dhia, A.-S. B., 2013. "On the numerical computation of nonlinear normal modes for reduced-order modelling of conservative vibratory systems". Mechanical Systems and Signal Processing, 36 (2), pp. 520-539.

[44] Wang, Y., Palacios, R., and Wynn, A., 2015. "A method for normal-mode-based model reduction in nonlinear dynamics of slender structures". Computers \& Structures, 159, pp. 26-40.

[45] Amabili, M., Sarkar, A., and Pardoussis, M., 2003. "Reduced-order models for nonlinear vibrations of cylindrical shells via the proper orthogonal decomposition method". Journal of Fluids and Structures, 18 (2), pp. 227-250.

[46] Kerschen, G., Feeny, B., and Golinval, J.-C., 2003. "On the exploitation of chaos to build reduced-order models". Computer Methods in Applied Mechanics and Engineering, 192 (13) , pp. $1785-1795$.

[47] Vakakis, A. F., Gendelman, O. V., Bergman, L. A., McFarland, D. M., Kerschen, G., and Lee, Y. S., 2008. Nonlinear targeted energy transfer in mechanical and structural systems, vol. 156. Springer Science \& Business Media.

[48] Kuether, R. J., Deaner, B. J., Hollkamp, J. J., and Allen, M. S., 2015. "Evaluation of geometrically nonlinear reduced-order models with nonlinear normal modes". AIAA Journal, pp. $1-13$.

[49] Wang, X., 2010. "Construction of frequency-energy plots for nonlinear dynamical systems from time-series data".

[50] Peter, S., Grundler, A., Reuss, P., Gaul, L., and Leine, R. I., 2016. "Towards finite element model updating based on nonlinear normal modes". In Nonlinear Dynamics, Volume 1. Springer, pp. 209-217.

[51] Vakakis, A. F., Manevitch, L. I., Mikhlin, Y. V., Pilipchuk, V. N., and Zevin, A. A., 1996. Normal modes and localization in nonlinear systems. Springer.

[52] Ilbeigi, S., and Chelidze, D., 2016. "Reduced order models for systems with disparate spatial and temporal scales". In Rotating Machinery, Hybrid Test Methods, Vibro-Acoustics $\&$ Laser Vibrometry, Volume 8. Springer, pp. 447-455.

[53] Chelidze, D., 2014. "Identifying robust subspaces for dynamically consistent reduced-order models". In Nonlinear Dynamics, Volume 2. Springer, pp. 123-130.

[54] Chelidze, D., and Zhou, W., 2006. "Smooth orthogonal decomposition-based vibration mode identification". Journal of Sound and Vibration, 292 (3), pp. 461-473.

[55] Sauer, T., Yorke, J. A., and Casdagli, M., 1991. "Embedology". Journal of statistical Physics, 65 (3-4), pp. 579-616.

[56] Kennel, M. B., Brown, R., and Abarbanel, H. D., 1992. "Determining embedding dimension for phase-space reconstruction using a geometrical construction". Physical review A, 45 (6), p. 3403 . 


\section{Appendix A: Governing equation of mass-spring-grid system}

$$
\begin{aligned}
& \begin{cases}m_{i} \ddot{x}_{i}+\left(c_{i}+c_{i+1}\right) \dot{x}_{i}-c_{i+1} \dot{x}_{i+1}+\left(k_{i}+k_{i+1}\right) x_{i}- & \\
k_{i+1} x_{i+1}+\left(k_{i}-k_{i+1}\right) a-k_{i} \frac{l}{l i}\left(a+\Delta x_{i}\right)+ & \text { for } i=1 \\
k_{i+1} \frac{l}{l_{i+1}}\left(a+\Delta x_{i+1}\right)=0 & \\
m_{i} \ddot{x}_{i}-c_{i} \dot{x}_{i-1}+\left(c_{i}+c_{i+1}\right) \dot{x}_{i}-c_{i+1} \dot{x}_{i+1}-k_{i} x_{i-1}+ & \\
\left(k_{i}+k_{i+1}\right) x_{i}-k_{i+1} x_{i+1}+\left(k_{i}-k_{i+1}\right) a- & \text { for } 2 \leq i \leq n-1 \\
k_{i} \frac{l}{l i}\left(a+\Delta x_{i}\right)+k_{i+1} \frac{l}{l_{i+1}}\left(a+\Delta x_{i+1}\right)=0 & \\
m_{i} \ddot{x}_{i}-c_{i} \dot{x}_{i-1}+\left(c_{i}+c_{i+1}\right) \dot{x}_{i}-k_{i} x_{i-1}+ & \\
\left(k_{i}+k_{i+1}\right) x_{i}+\left(k_{i}-k_{i+1}\right) a-k_{i} \frac{l}{l i}\left(a+\Delta x_{i}\right)+ & \\
k_{i+1} \frac{l}{l_{i+1}}\left(a+\Delta x_{i+1}\right)=0 & \text { for } i=n \\
m_{i} \ddot{y}_{i}+\left(c_{i}+c_{i+1}\right) \dot{y}_{i}-c_{i+1} \dot{y}_{i+1}+\left(k_{i}+k_{i+1}\right) y_{i}- & \\
k_{i+1} y_{i+1}-k_{i} \frac{l}{l i} \Delta y_{i}+k_{i+1} \frac{l}{l_{i+1}} \Delta y_{i+1}=F_{y, i}(t) & \text { for } i=1 \\
m_{i} \ddot{y}_{i}-c_{i} \dot{y}_{i-1}+\left(c_{i}+c_{i+1}\right) \dot{y}_{i}-c_{i+1} \dot{y}_{i+1}-k_{i} y_{i-1}+ & \\
\left(k_{i}+k_{i+1}\right) y_{i}-k_{i+1} y_{i+1}-k_{i} \frac{l}{l i} \Delta y_{i}+ & \text { for } 2 \leq i \leq n-1 \\
k_{i+1} \frac{l}{l_{i+1}} \Delta y_{i+1}=F_{y, i}(t) & \text { for } i=n \\
m_{i} \ddot{y}_{i}-c_{i} \dot{y}_{i-1}+\left(c_{i}+c_{i+1}\right) \dot{y}_{i}-k_{i} y_{i-1}+ \\
\left(k_{i}+k_{i+1}\right) y_{i}-k_{i} \frac{l}{l i} \Delta y_{i}+k_{i+1} \frac{l}{l_{i+1}} \Delta y_{i+1}=F_{y, i}(t)\end{cases}
\end{aligned}
$$

540

$$
\Delta x_{i}= \begin{cases}x_{i} & \text { for } i=1 \\ x_{i}-x_{i-1} & \text { for } 2 \leq i \leq n \\ -x_{i-1} & \text { for } i=n+1\end{cases}
$$

$$
\Delta y_{i}= \begin{cases}y_{i} & \text { for } i=1 \\ y_{i}-y_{i-1} & \text { for } 2 \leq i \leq n \\ -y_{i-1} & \text { for } i=n+1\end{cases}
$$

${ }_{541}$ We should note that here only linear damping is considered for the system. 